\title{
STOCHASTIC MODELING AND SENSITIVITY ANALYSIS OF INTEGRATED HARDWARE-SOFTWARE SYSTEM SUBJECT TO VARYING WEATHER CONDITIONS
}

\author{
Ashish Kumar'a and Monika Saini' ${ }^{2 a^{*}}$ \\ aDepartment of Mathematics \& Statistics, Manipal University Jaipur, Jaipur-303007, Rajasthan, INDIA. Email: \\ akbrk@rediffmail.com ${ }^{1}$; drmnksaini4@gmail.com² \\ *Corresponding author: drmnksaini4@gmail.com \\ Received: $25^{\text {th }}$ Sep $2019 \quad$ Accepted: $17^{\text {th }}$ May 2021 \\ DOI: https://doi.org/10.22452/mjs.vol40no3.5 \\ Published: $31^{\text {st }}$ Oct 2021
}

\begin{abstract}
This study aims to provide a stochastic investigation of an integrated hardware-software system considering hardware failure, software up-gradation upon failure, precautionary maintenance (PM) after a pre-determined process time, maximum repair time of hardware and varying weather conditions. All time dependent indiscriminate variables were arbitrarily dispersed. Some prominent reliability measures such as mean time to system failure (MTSF), availability and revenue of the system were obtained using a well-established semiMarkov process. The regenerative point technique has also been taken into consideration during model development. Sensitivity analysis of these measures was also performed. Finally, empirical analysis was carried out to demonstrate the results for a specific case. To highlight the significance of the study, MTSF graphs, availability, profit and sensitivity were also depicted.
\end{abstract}

Keywords: Integrated Hardware-Software System, Hardware failure, Software up-gradation, Weather conditions and Precautionary Maintenance

\section{INTRODUCTION}

Numerous studies such as those by Friedman and Tran (1992), Welke et al. (1995), and Lai et al. (2002) have been carried out on the stochastic analysis of two-unit cold standby integrated hardware and software, i.e., computer systems underneath a diverse set of conventions. Malik and Nandal (2010) used the concept of priority and maximum operation time in cold standby redundant systems. Malik and Anand (2010), Kumar et al. (2012) and Kumar and Malik (2011, 2012, 2014) proposed several stochastic models for computer systems with different sets of assumptions. However, they did not consider the economic factor; 60\%-70\% population cannot afford standby systems. Many authors such as Kumar and Saini
(2014) and Barak and Barak (2016) have studied single-unit computer systems by taking either hardware or software components individually in standby. Barak et al. (2014) developed a reliability model for a single unit system under abnormal weather conditions. In these studies, no attention was given to precautionary maintenance and pre-determined process time specified for hardware repair in advance. In addition, all of these studies assume that the conditions specified for system operation remain homogenous during the entire period, and follow constant failure and repair rates. These homogenous conditions are also called weather conditions. Some researchers such as Malik and Barak (2007) and Malik and Pawar (2010) attempted to analyze the effect of weather conditions on single unit 
systems. Due to excessive use, however, the failure rate does not remain constant. The ambition of this current study is to analyze a single-unit integrated hardware and software system subject to hardware failure, software failure, precautionary maintenance, pre-determined process time, arbitrary distribution for failure and repair rates and weather conditions. Unambiguous expressions for the resulting reliability characteristics of the system were obtained for a particular case, by considering all failure and repair rates as exponential distributed. Sensitivity analysis of mean time to system failure (MTSF) and availability of the system were also performed. Mobile phones, laptops and desktops may be taken as factual illustrations for the system model discussed in this study. The excessive use of all these systems results in the reduction in efficiency and working capacity.

\section{Assumptions}

1. The system consists of one unit in which hardware and software machineries are organized.

2. Hardware and software failures are independent.

3. No two types of failures occurs simultaneously.

4. The unit endures precautionary maintenance after completion of a pre-determined process time.

5. The replacement of the failed hardware component after maximum repair time is not affected by weather conditions.

6. Repair and preventive maintenance procedures are perfect.

7. There is one repairman.

8. The switch devices are perfect.

\title{
Notations and states of the system
}

\author{
$E_{i}(t)(i=1,2)$ : Represents cumulative density function for hardware and \\ software components respectively. \\ $H(t)$ : Cumulative distribution function (cdf) of preventive \\ maintenance rate \\ $H_{1}(t)$ : cdf of pre-determined process time prior to precautionary \\ maintenance \\ $M(t)$ : cdf of changeover rate after abnormal to normal weather \\ conditions \\ $M_{1}(t)$ : cdf of changeover rate after normal to abnormal weather \\ conditions \\ $K(t)$ : cdf of maximum repair time \\ $L(t)$ : cdf of replacement time \\ $G(t)$ : Represents hardware repair rate's cdf \\ $F(t)$ : Represents software up-gradation rate's cdf \\ $O$ : Operative state \\ SFup : Unit suffers due to software botch and under up-gradation \\ HFur: Unit suffers due to hardware botch and under repair \\ HFurp : Hardware component is under replacement after maximum \\ repair time \\ $P m$ : Unit under precautionary maintenance after pre-determined \\ process time \\ $\bar{O}, \overline{H F w r}, \overline{W P m}, \overline{S F w u p}$ : Represents abnormal weather affected states. In these states, all \\ repair or operational activities are paused
}


$U:$ Set of regenerative states

$\Pi_{i}(t): \quad \mathrm{P}$ [system remains in upstate at time $\mathrm{t} / S_{i} \in U$ ]

$B_{i}(t): \quad \mathrm{P}$ [repairman is busy in repair activities at time $\mathrm{t}$, starting from $\left.S_{i} \in U\right]$

$Z_{i}(t): \quad \mathrm{p}$ [system is up primarily at $S_{i} \in U$ up to time t deprived of moving to any other regenerative state, including self-state]

(c): Symbol for convolution

$u$ : Dummy variable

$S_{i}$ : Represents system states

Considering the above notations, the system can be in any one of the resulting states:

$S_{0}=O S_{1}=$ SFup $S_{2}=H F u r S_{3}=\bar{O} S_{4}=\overline{H F w r} S_{5}=H F u r p S_{6}=P m S_{7}=\overline{W P m} S_{8}=\overline{S F w u p}$

Transition probabilities and sojourn times

By using the fundamentals concepts of the probability theory, the instants of visiting into any states $S_{i} \in U$ are regenerative points. Let $U_{0}(\equiv 0), U_{1}, U_{2} \ldots$. denote the instants which the system reaches at any state $S_{i} \in U$. Let $X_{n}$ represent the state at instant $U_{n+}$, i.e., after transition at $U_{n}$. Then $\left(X_{n}, U_{n}\right)$ and $Q_{i j}(t)=P\left[X_{n+1}=j, U_{n+1}-U_{n} \leq t / X_{n}=i\right]$ are termed as the Markov-renewal process and the semi-Markov kernel over U respectively. Assume that $P=\left(p_{i j}\right)=\left(Q_{i j}(\infty)=Q(\infty)\right)$ represents the t.p.m. of the embedded Markov chain having some zero elements. By probability theoretic concepts, the non-zero elements $p_{i j}$ are

$$
p_{01}=\int \overline{E_{1}}(t) \overline{M_{1}}(t) \overline{H_{1}}(t) d E_{2}(t)
$$

where $p_{01}$ means the system does not face any type of hardware failure until time t, PM does not attain until time $t$, weather does not become abnormal until time $t$, but software fails and unit is under upgradation. The rest of the transition probabilities can be derived in a similar fashion.

$$
\begin{aligned}
& p_{02}=\int \overline{E_{2}}(t) \overline{M_{1}}(t) \overline{H_{1}}(t) d E_{1}(t) ; \quad p_{03}=\int \overline{E_{1}}(t) \overline{E_{2}}(t) \overline{H_{1}}(t) d M_{1}(t) ; \\
& p_{06}=\int \overline{E_{1}}(t) \overline{M_{1}}(t) \overline{E_{2}}(t) d H_{1}(t) \quad p_{10}=\int \overline{M_{1}}(t) d F(t) ; \quad p_{18}=\int \bar{F}(t) d M_{1}(t) ; \\
& p_{20}=\int \overline{M_{1}}(t) \bar{K}(t) d G(t) ; \quad p_{25}=\int \bar{G}(t) \overline{M_{1}}(t) d K(t) \\
& p_{24}=\int \bar{G}(t) \bar{K}(t) d M_{1}(t) ; \quad p_{30}=\int d M(t) ; p_{42}=\int d M(t) ; p_{50}=\int d L(t) ; p_{67}=\int \bar{H}(t) d M_{1}(t) ; \\
& p_{60}=\int \overline{M_{1}}(t) d H(t) ; p_{76}=\int d M(t) ; p_{81}=\int d M(t)
\end{aligned}
$$


One can easily verified that:

$$
\begin{array}{llll}
p_{01}+p_{02}+p_{03}+p_{06}=1 & p_{10}+p_{18}=1 & p_{20}+p_{24}+p_{25}=1 & p_{30}=1 \\
p_{50}=1 & p_{60}+p_{67}=1 & p_{76}=1 & p_{81}=1
\end{array} \quad p_{42}=1
$$

The mean sojourn times $\psi_{i}$ at state $S_{i} \in U$ are:

$$
\begin{aligned}
& \psi_{0}=\int \overline{E_{1}}(t) \overline{E_{2}}(t) \overline{M_{1}}(t) \overline{H_{1}}(t) d(t) ; \psi_{1}=\int \overline{M_{1}}(t) \bar{F}(t) d(t) ; \psi_{2}=\int \bar{G}(t) \overline{M_{1}}(t) \bar{K}(t) d(t) \\
& \psi_{3}=\int \bar{M}(t) d(t) \psi_{4}=\int \bar{M} d(t) \psi_{5}=\int \bar{L} d(t) \psi_{6}=\int \overline{M_{1}}(t) \bar{H}(t) d(t) ; \psi_{7}=\int \bar{M}(t) d(t) \\
& \psi_{8}=\int \bar{M}(t) d(t)
\end{aligned}
$$

\section{Mean Time to System Failure}

By using a semi-Markov approach and regenerative processes, we obtained the following relations:

$$
\Psi_{0}(t)=\overline{E_{1}}(t) \overline{E_{2}}(t) \overline{M_{1}}(t) \overline{H_{1}}(t)
$$

Equation (1) can be described as follows: the L.H.S. term means that the system works until time $t$, while the R.H.S. term means that hardware and software failure, pre-determined process time and maximum repair time do not occur until time t.

$$
M T S F=E(T)=\frac{1}{\left(a \lambda_{1}+b \lambda_{2}+\alpha_{0}+\beta_{0}\right)}
$$

\section{Availability analysis}

Making use of fundamental concepts inherent in the probability theory, we acquire resulting recurrence relations for $\prod_{i}(t), i \in U$, where:

$$
\begin{aligned}
& \Pi_{0}(t)=Z_{0}(t)+q_{01}(t) \odot \Pi_{1}(t)+q_{02}(t) \odot \Pi_{2}(t)+q_{03}(t) \odot \Pi_{3}(t)+q_{06}(t) \odot \prod_{6}(t) \\
& \Pi_{1}(t)=q_{10}(t) \odot \Pi_{0}(t)+q_{18}(t) \odot \prod_{8}(t) \\
& \Pi_{2}(t)=q_{20}(t) \odot \Pi_{0}(t)+q_{24}(t) \odot \Pi_{4}(t)+q_{25}(t) \odot \Pi_{5}(t) \\
& \Pi_{3}(t)=q_{30}(t) \odot \prod_{0}(t) ; \Pi_{4}(t)=q_{42}(t) \odot \Pi_{2}(t) ; \Pi_{5}(t)=q_{50}(t) \odot \Pi_{0}(t) \\
& \Pi_{6}(t)=q_{60}(t) \odot \Pi_{0}(t)+q_{67}(t) \odot \Pi_{7}(t) \\
& \Pi_{7}(t)=q_{76}(t) \odot \prod_{6}(t) ; \Pi_{8}(t)=q_{81}(t) \odot \Pi_{1}(t)
\end{aligned}
$$

where:

$$
Z_{0}(t)=\overline{E_{1}}(t) \overline{E_{2}}(t) \overline{M_{1}}(t) \overline{H_{1}}(t)
$$


Taking the Laplace Transforms of (3) to elucidate them for $\Pi_{0}^{*}(u)$, we get availability of the system for $\prod_{0}$ in the form of:

$$
\prod_{0}=\lim _{t \rightarrow 0} \prod_{0}(t)=\lim _{u \rightarrow 0} u \prod_{0}^{*}(u)=\frac{n_{1}}{N_{1}}
$$

where $n_{1}=\lambda \gamma \alpha \tau_{1}\left(\beta+\eta_{0}\right)$

and

$$
\begin{aligned}
N_{1} & =\left(\alpha+\beta_{0}\right)\left(\beta+\eta_{0}\right) \lambda \gamma \tau_{1}+\left(\alpha+\beta_{0}\right)\left(\beta+\eta_{0}\right) b \lambda_{2} \gamma \tau_{1}+a \lambda_{1} \lambda \tau_{1}\left(\alpha \tau_{1}+\eta_{0} \alpha+\beta_{0} \tau_{1}\right) \\
& +\alpha_{0} \lambda \tau_{1}\left(\alpha+\beta_{0}\right)\left(\beta+\eta_{0}\right)
\end{aligned}
$$

\section{Busy period analysis}

Making use of fundamental concepts of the probability theory, we achieve the resulting recurrence relations for $B_{i}(t), i \in U$, where:

$$
\begin{aligned}
& B_{0}(t)=q_{01}(t) \odot B_{1}(t)+q_{02}(t) \odot B_{2}(t)+q_{03}(t) \odot B_{3}(t)+q_{06}(t) \odot B_{6}(t) \\
& B_{1}(t)=Z_{1}(t)+q_{10}(t) \odot B_{0}(t)+q_{18}(t) \odot B_{8}(t) \\
& B_{2}(t)=Z_{2}(t)+q_{20}(t) \oplus B_{0}(t)+q_{24}(t) \oplus B_{4}(t)+q_{25}(t) \odot B_{5}(t) \\
& B_{3}(t)=q_{30}(t) \odot B_{0}(t) ; B_{4}(t)=q_{42}(t) \odot B_{2}(t) ; B_{5}(t)=Z_{5}(t)+q_{50}(t) \odot B_{0}(t) \\
& B_{6}(t)=Z_{6}(t)+q_{60}(t) \odot B_{0}(t)+q_{67}(t) \odot B_{7}(t) \\
& B_{7}(t)=q_{76}(t) \odot B_{6}(t) ; \prod_{8}(t)=q_{81}(t) \odot \prod_{1}(t)
\end{aligned}
$$

where:

$$
Z_{1}(t)=\overline{M_{1}}(t) \bar{F}(t) ; Z_{2}(t)=\bar{G}(t) \overline{M_{1}}(t) \bar{K}(t) ; Z_{5}(t)=\bar{L}(t) ; Z_{5}(t)=\overline{M_{1}}(t) \bar{H}(t)
$$

Taking the Laplace Transforms of (4) for $B_{0}^{*}(u)$, we get a busy period by the server due to hardware repair, software up-

$$
B_{0}=\lim _{t \rightarrow 0} B_{0}(t)=\lim _{u \rightarrow 0} u B_{0}^{*}(u)=\frac{n_{2}}{N_{1}}
$$

where:

$$
\begin{aligned}
n_{2} & =\alpha\left(a \lambda_{1}+b \lambda_{2}+\alpha_{0}+\beta_{0}\right)\left[\alpha_{0} \lambda \tau_{1}\left(a \lambda_{1}+b \lambda_{2}+\alpha_{0}+\beta_{0}\right)\left(\beta+\eta_{0}\right)+\left(\beta+\eta_{0}\right)\left(\lambda+\beta_{0}\right) b \lambda_{2} \tau_{1} \gamma\right. \\
& +\left(a \lambda_{1}+b \lambda_{2}+\alpha_{0}+\beta_{0}\right) a \lambda_{1} \lambda\left(\gamma+\beta_{0}\right)\left(\tau_{1}+\eta_{0}\right)
\end{aligned}
$$

and

$$
\begin{aligned}
N_{1} & =\left(\alpha+\beta_{0}\right)\left(\beta+\eta_{0}\right) \lambda \gamma \tau_{1}+\left(\alpha+\beta_{0}\right)\left(\beta+\eta_{0}\right) b \lambda_{2} \gamma \tau_{1}+a \lambda_{1} \lambda \tau_{1}\left(\alpha \tau_{1}+\eta_{0} \alpha+\beta_{0} \tau_{1}\right) \\
& +\alpha_{0} \lambda \tau_{1}\left(\alpha+\beta_{0}\right)\left(\beta+\eta_{0}\right)
\end{aligned}
$$




\section{Cost-benefit Analysis}

Since

$$
C(t)=\delta \mu_{u p}(t)-\mu \mu_{b}(t)
$$

where:

$\delta:$ Income per unit time.

$\mu$ : Cost per unit time of repair by server due to $\mathrm{h} / \mathrm{w}$ repair, s/w up-gradation, $\mathrm{PM}$, and replacement.

$$
\begin{gathered}
\mu_{u p}(t)=\int_{0}^{t} \Pi_{0}(t) \\
\mu_{b}(t)=\int_{0}^{t} B_{0}(t) \\
C=\lim _{t \rightarrow 0} \frac{C(t)}{t}
\end{gathered}
$$

\section{Sensitivity Analysis}

The sensitivity of reliability measures is a demanding input factor defined in terms of the partial derivative of reliability measures, with respect to failure rates. An effort has been made to perform sensitivity analysis for changes in MTSF, steady sate availability and profit of the system, resulting in changes in various parameters. By differentiating Equation (2), we achieve

$$
\frac{\partial(M T S F)}{\partial \lambda_{1}}, \frac{\partial(M T S F)}{\partial \lambda_{2}}, \frac{\partial(M T S F)}{\partial \alpha_{0}} \& \frac{\partial(M T S F)}{\partial \beta_{0}} .
$$

In a similar fashion, we obtain the sensitivity expression for steady state availability, as follows:

$$
\frac{\partial\left(\Pi_{0}\right)}{\partial \lambda_{1}}, \frac{\partial\left(\Pi_{0}\right)}{\partial \lambda_{2}}, \frac{\partial\left(\Pi_{0}\right)}{\partial \alpha_{0}} \& \frac{\partial\left(\Pi_{0}\right)}{\partial \beta_{0}}
$$

where:

$$
\begin{aligned}
& \frac{\partial(M T S F)}{\partial \lambda_{1}}=\frac{-a}{\left(a \lambda_{1}+b \lambda_{2}+\alpha_{0}+\beta_{0}\right)^{2}} ; \\
& \frac{\partial(M T S F)}{\partial \lambda_{2}}=\frac{-b}{\left(a \lambda_{1}+b \lambda_{2}+\alpha_{0}+\beta_{0}\right)^{2}} \\
& \frac{\partial(\text { MTSF })}{\partial \beta_{0}}=\frac{-1}{\left(a \lambda_{1}+b \lambda_{2}+\alpha_{0}+\beta_{0}\right)^{2}} \\
& \frac{\partial\left(\prod_{0}\right)}{\partial \lambda_{1}}=\frac{-n\left(\alpha \tau_{1}+\alpha \eta_{0}+\beta_{0} \tau_{1}\right) a \lambda \gamma}{N^{2}} ; \quad \frac{\partial\left(\prod_{0}\right)}{\partial \lambda_{2}}=\frac{-n\left(\alpha+\beta_{0}\right)\left(\eta_{0}+\beta\right) b \gamma \tau_{1}}{N^{2}} \\
& ; \frac{\partial\left(\prod_{0}\right)}{\partial \beta_{0}}=\frac{-n\left\{\left(\eta_{0}+\beta\right) \lambda \tau_{1} \gamma+\left(\eta_{0}+\beta\right) b \lambda_{2} \tau_{1} \gamma+a \lambda_{1} \lambda \tau_{1} \gamma+\alpha_{0}\left(\eta_{0}+\beta\right) \lambda \tau_{1}\right\}}{N^{2}}
\end{aligned}
$$




\section{Numerical Analysis:}

This section depicts the graphical results of mean time to system failure, availability, profit, sensitivity analysis of MTSF and sensitivity analysis of availability, by considering all repair and failure rates as exponentially distributed as

$$
\begin{aligned}
& e_{1}(t)=a \lambda_{1} e^{-a \lambda_{1} t}, e_{2}(t)=b \lambda_{2} e^{-b \lambda_{2} t}, m(t)=\alpha e^{-\alpha t}, f(t)=\lambda e^{-\lambda t}, g(t)=\beta e^{-\beta t}, m_{1}(t)=\beta_{0} e^{-\beta_{0} t}, \\
& l(t)=\tau_{1} e^{-\tau_{1} t}, h_{1}(t)=\alpha_{0} e^{-\alpha_{0} t}, h(t)=\gamma e^{-\gamma t}, k(t)=\eta_{0} e^{-\eta_{0} t} .
\end{aligned}
$$

The following arbitrary values and costs are assigned to various parameters, as follows:

$$
\begin{aligned}
\text { Series }-1: a & =0.7, b=0.3, \lambda_{1}=(0.01-0.1), \lambda_{2}=0.05, \lambda=0.9, \alpha_{0}=5, \eta_{0}=30, \gamma=30, \beta_{0}=0.03, \\
\alpha & =0.93, \tau_{1}=0.34 \\
\text { Series }-2: a & =0.7, b=0.3, \lambda_{1}=(0.01-0.1), \lambda_{2}=0.2, \lambda=0.9, \alpha_{0}=5, \eta_{0}=30, \gamma=30, \beta_{0}=0.03, \\
\alpha & =0.93, \tau_{1}=0.34 \\
\text { Series }-3: a & =0.7, b=0.3, \lambda_{1}=(0.01-0.1), \lambda_{2}=0.05, \lambda=0.9, \alpha_{0}=5, \eta_{0}=30, \gamma=30, \beta_{0}=0.8, \\
\alpha & =0.93, \tau_{1}=0.34 \\
\text { Series }-4: a & =0.3, b=0.7, \lambda_{1}=(0.01-0.1), \lambda_{2}=0.05, \lambda=0.9, \alpha_{0}=5, \eta_{0}=30, \gamma=30, \beta_{0}=0.03, \\
\alpha & =0.93, \tau_{1}=0.34
\end{aligned}
$$

The income produced by the system per unit up time is considered $\delta=6000$, and spending on repair activities is $\mu=500$. The sensitivity analysis of MTSF and availability was also performed by changing the values of hardware failure rate $\left(\lambda_{1}\right)$, software failure rate $\left(\lambda_{2}\right)$ and abnormal weather conditions $\left(\beta_{0}\right)$, between 0.01-0.1.

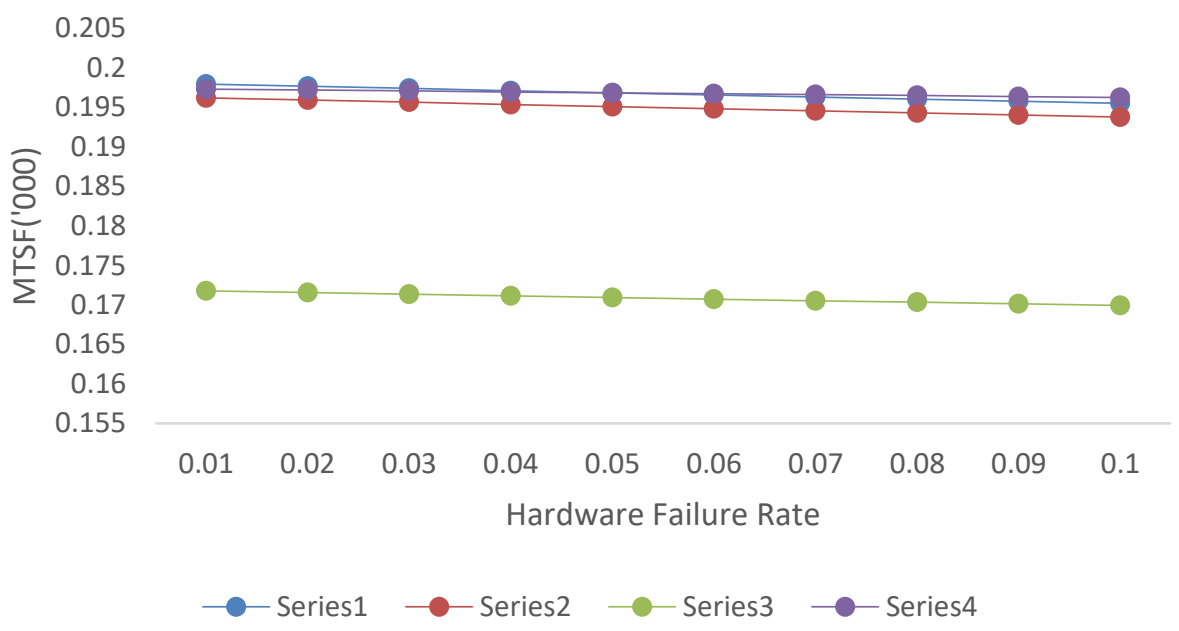

Figure 1. MTSF Vs. Hardware Failure rate 


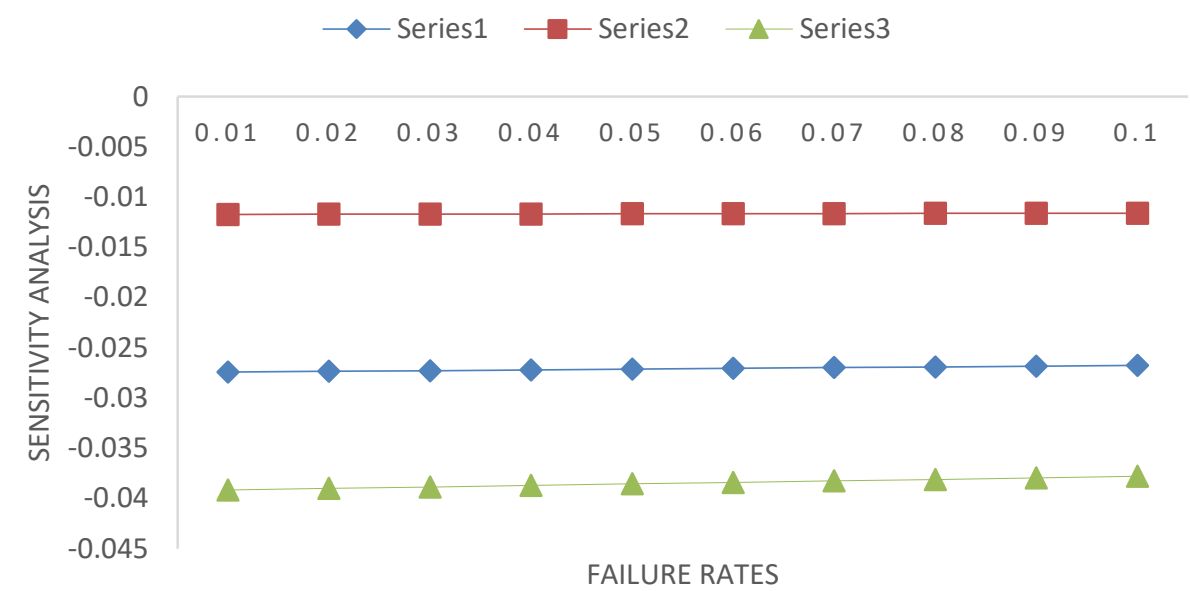

Figure 2. Sensitivity Analysis of MTSF vs. Various Failure Rates

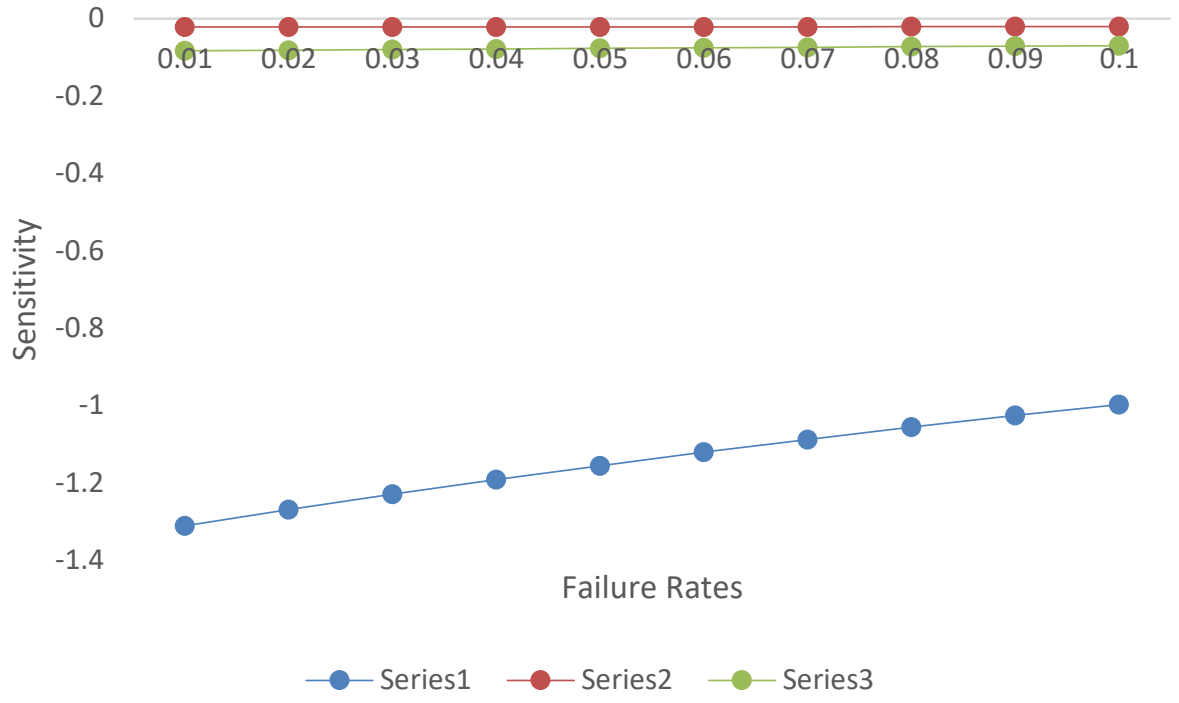

Figure 3. Sensitivity Analysis of Availability vs. Various Failure Rates

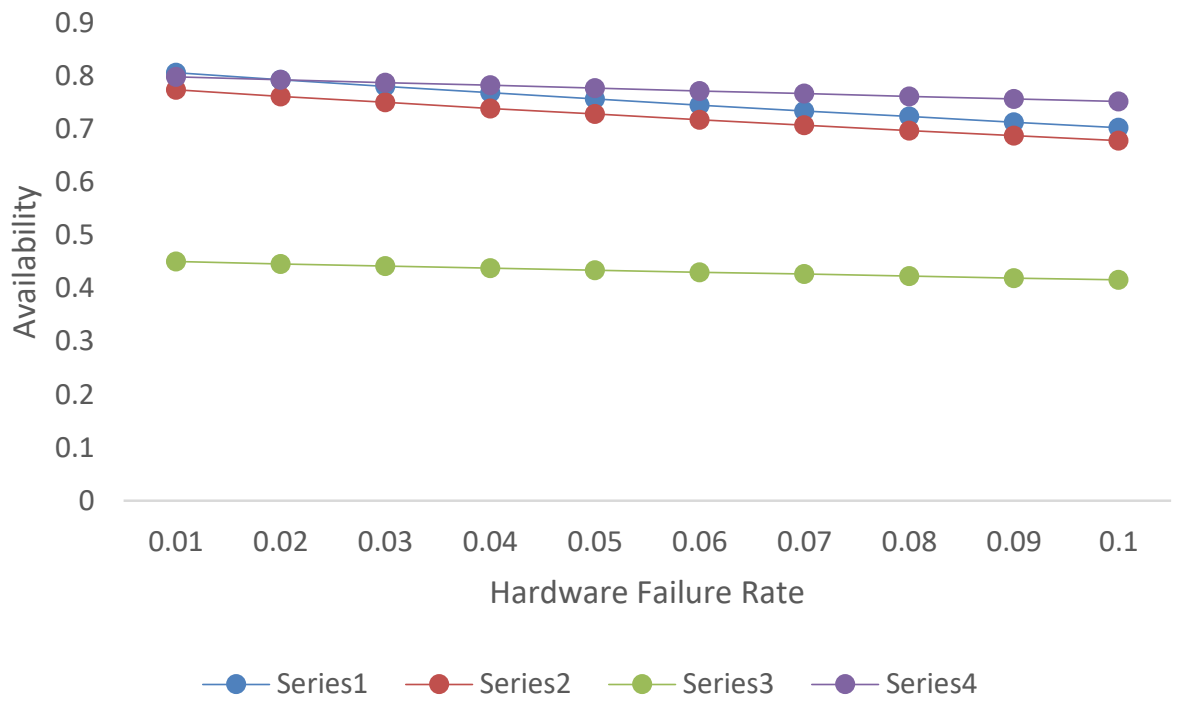

Figure 4. Availability vs. Various Failure Rates 


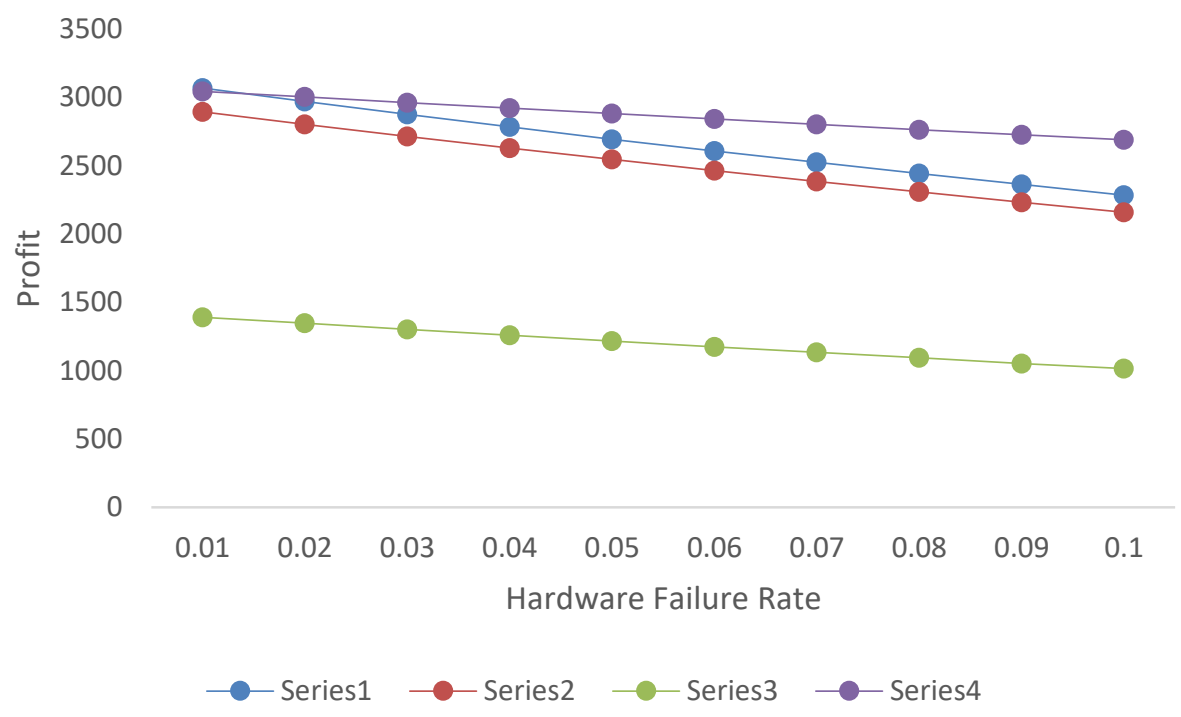

Figure 5. Profit vs. Various Failure Rates

\section{CONCLUSION}

According to the graphical results shown in Fig. 1-5, the mean time to system failure, availability and revenue of the integrated $\mathrm{h} / \mathrm{w}$ and $\mathrm{s} / \mathrm{w}$ system rapidly decrease with the surge of the hardware and software failure rates, maximum operation time, maximum repair time and abnormal weather conditions rate. From Figs. 2 and 3, MTSF is more sensitive with respect to abnormal weather conditions, while availability is most affected by the variation in the failure rate of hardware rate. If the chance of software failure increases, the system becomes less available and profitable. Finally, it is concluded that an integrated $\mathrm{h} / \mathrm{w}$ and $\mathrm{s} / \mathrm{w}$ system can be made more consistent and cost-effective by controlling the software failure rate and abnormal weather conditions.

\section{ACKNOWLEDGEMENT}

Authors are very thankful to reviewers for giving valuables suggestions to enhance quality of manuscript.

\section{REFERENCES}

Barak, A.K. \& Barak, M.S. (2016). Impact of Abnormal Weather Conditions on Various Reliability Measures of a Repairable System with Inspection. Thailand Statistician, 14(1), 35-45.

Barak, A.K., Barak, M.S. \& Malik, S.C. (2014) Reliability Analysis of a Single-Unit System with Inspection Subject to Different Weather Condition., Journal of Statistics and Management Systems, 17:2, 195206.

Friedman, M. A., \& Tran, P. (1992). Reliability techniques for combined hardware / software systems. Proceedings of Annual Reliability and Maintainability Symposium. 209-293.

Kumar, A \& Malik, S.C. (2012). Stochastic modeling of a computer system with priority to $\mathrm{PM}$ over $\mathrm{S} / \mathrm{W}$ replacement subject to maximum operation and repair times. International Journal of Computer Applications. 43(3), 27-34. 
Kumar, A. \& Malik, S. C. (2014). Reliability Modelling of a Computer System with Priority to H/W Repair over Replacement of $\mathrm{H} / \mathrm{W}$ and Up-gradation of S/W Subject to MOT and MRT. Jordan Journal of Mechanical and Industrial Engineering. 8(4), 233241.

Kumar, A. \& Malik, S.C. (2011). Profit analysis of a computer system with priority to software replacement over hardware repair subject to maximum operation and repair times, International Journal of Engineering Science and Technology. 3(10), 7452-7468.

Kumar, A. \& Saini, M. (2014). CostBenefit Analysis of a Single-Unit System with Preventive Maintenance and Weibull Distribution for Failure and Repair Activities. Journal of Applied Mathematics, Statistics and Informatics. 10(2), 5-19.

Kumar, A., Malik, S. C., \& Barak, M. S. (2012). Reliability Modelling of a Computer System with Independent H/W and S/W Failures Subject to Maximum Operation and Repair Times. International Journal of Mathematical Archive, 3(7).

Lai, C. D., M. Xie, Poh, K. L., Dai, Y. S., \& Yang, P. (2002). A model for availability analysis of distributed software / hardware systems.
Information and Software Technology, 44, 343-350.

Malik, S. C. \& Anand, J. (2010). Reliability and Economic Analysis of a Computer System with Independent Hardware and Software Failures. Bulletin of Pure and Applied Sciences, 29E(1), 141-153.

Malik, S.C \& Nandal, P. (2010). CostAnalysis of Stochastic Models with Priority to Repair Over Preventive Maintenance Subject to Maximum Operation Time, Edited Book, Learning Manual on Modeling, Optimization and Their Applications, Excel India Publishers, 165-178.

Malik, S.C. \& Barak, M.S. (2007). Probabilistic analysis of a singleserver system operating under different weather conditions. Journal of Mathematical Analysis and Approximation Theory. 2(2), 173-182.

Malik, S.C. \& Pawar, D. (2010). Reliability and economic measures of a system with inspection for on-line repair and no repair activity in abnormal weather. Bulletin of Pure and Applied Sciences. 29 E (2), 355368.

Welke, S. R., Johnson, B. W., \& Aylar, J. H. (1995). Reliability modeling of hardware / software systems. IEEE Transactions on Reliability. 44(3), 413-418. 